\title{
Étude des caractéristiques projectives des objets spatiaux et de leurs relations
}

\author{
Roland Billen* - Eliseo Clementini** \\ * Department of Geography and Geomatics, Center for Geosciences, University of \\ Glasgow, Glasgow G12 8QQ, Royaume-Uni \\ rbillen@geog.gla.ac.uk \\ ** Dipartimento di Ingegneria Elettrica, Università degli Studi di L’Aquila, Poggio di \\ Roio, 67040 L'Aquila, Italie \\ eliseo@ing.univaq.it
}

RÉSUMÉ. L'espace projectif a été peu exploré dans la caractérisation des objets spatiaux et de leurs relations. Ce travail présente les résultats d'une démarche "projective » concernant la forme des objets spatiaux et les relations binaires et ternaires entre objets. En offrant un cadre mathématique formel à une description qualitative de la forme des objets ainsi qu'à des relations telles que "l'objet $A$ touche un coin de l'objet $B$ » ou bien «l'objet $A$ se trouve à gauche des objets $B$ et $C$ », les modèles présentés se révèlent d'un grand intérêt dans le développement de l'ontologie spatiale.

ABSTRACT. The projective space has been poorly explored for the characterisation of spatial objets and their relationships. This work presents the results of a "projective » approach about spatial objects shapes and about binary and ternary relationships between objects. The presented models are of prime interest for the development of spatial ontology. They provide a formal mathematical framework allowing a qualitative description of the object's shapes as well as relationships such as "the object A touches a corner of the object B" or "the object $A$ is on the left side of the objects B and C".

MOTS-CLÉS : Relations spatiales, relations projectives, modèles qualitatifs, modèle dimensionnel, ontologie spatiale.

KEYWORDS: Spatial relations, projective relations, qualitative models, dimensional model, spatial ontology. 


\section{Introduction}

Un courant important de la recherche en Sciences de l’Information Géographique concerne l'étude des concepts fondamentaux régissant notre perception de la spatialité des objets. Cette tendance se traduit par un nombre important de travaux sur les ontologies spatiales et temporelles. Une des bases de notre connaissance en la matière repose sur la description de la géométrie des objets manipulés ainsi que l'identification des relations spatiales existantes entre ces objets. Depuis plus d'une décennie, les études concernant les caractéristiques topologiques des objets et de leurs relations ont mené à des progrès majeurs dans la compréhension des phénomènes spatiaux mais également dans la traduction de ces phénomènes à travers des modèles particuliers (Egenhofer et al., 1991 ; Clementini et al., 1993 ; Cui et al., 1993). Dans le même temps, d'autres modèles qualitatifs concernant les relations métriques de distance et de direction ont été développés (Freska, 1992 ; Hernandez, 1994 ; Clementini et al., 1997 ; Galton, 2000).

La vision topologique des objets mise en parallèle avec leur représentation dans un espace métrique a permis de définir des standards, des opérateurs, des structures de données largement implémentées dans les SIG et les SGBD spatiaux. Mais entre l'espace topologique et l'espace métrique, il existe d'autres espaces non-explorés ou si peu (Gapp, 1995 ; Vorwerg, 1997 ; Clementini et al., 2000). Nous soutenons l'idée que la prise en compte de ces espaces enrichit significativement le raisonnement spatial qualitatif et les ontologies spatiales. Nos recherches se basent sur l'étude des propriétés de l'espace projectif, un de ces espaces intermédiaires. L’approche consiste à considérer certaines caractéristiques de cet espace (ses invariants) dans le but de décrire les objets spatiaux et leurs relations. Ces descriptions doivent nous permettre de classer les objets suivant des critères qualitatifs concernant leur forme tels que la convexité ou les concavités, mais aussi de formaliser des relations telles que «l'objet A touche un coin de l'objet $B$ » ou bien «l'objet A aboutit sur une des arêtes de l'objet $\mathrm{B}$ », ou bien encore « l'objet $\mathrm{A}$ se trouve à gauche des objets $\mathrm{B}$ et $\mathrm{C}$ ». Notre objectif est de présenter notre approche dans sa globalité et son originalité, en évitant, quand une interprétation intuitive le permet, de décrire longuement les développements mathématiques issus de nos travaux.

L’article est organisé comme suit. D’abord, nous présentons brièvement le concept d'ontologie et d'ontologie spatiale dans le contexte de notre étude, ainsi que le champ d'application de nos travaux (cf. 2.). Puis, nous nous intéressons à la définition de la forme des objets sur base de critères projectifs ( $c f$. 3.). Dans cette partie, nous présentons d'abord le modèle dimensionnel ( $c$ f. 3.1.). Dans ce modèle, les objets spatiaux sont définis suivant des éléments constitutifs, appelés éléments dimensionnels, invariants sous des transformations projectives (Billen, 2002 ; Billen et al., 2002). Ensuite d'autres critères concernant la forme des objets sont envisagés, faisant référence à des travaux existants et prospectifs ( $c f$. 3.2.). Le deuxième grand volet de cet article concerne les relations projectives binaires ( $c f$. 4.). Sont abordées 
les relations spatiales exprimées sur base de l'étude des relations existantes entre les différents éléments dimensionnels des objets: les relations dimensionnelles. La troisième partie concerne l'étude des relations existantes entre trois objets dans un espace projectif (Billen et al. 2004) (cf. 5.). Les relations projectives ternaires ont été définies sur base d'une partition invariante de l'espace suivant des transformations projectives. Cette partition de l'espace se fait sur base de deux objets de référence, le troisième objet étant dit en relation ternaire avec les deux autres. Enfin, nous concluons l'étude en la replaçant dans le contexte de l'ontologie spatiale (cf. 6.).

\section{Contexte de l'étude - ontologie spatiale}

L'ontologie en tant que discipline philosophique a été redécouverte pour les besoins de l'Intelligence Artificielle (Hayes, 1978). La construction rigoureuse de modèles du monde réel implique que ce dont on parle soit fixé exactement et que les interactions soient spécifiées. En Intelligence Artificielle et en informatique, le terme ontologie réfère à un vocabulaire ou un système de classification qui décrit les concepts opérant dans un domaine particulier à travers des définitions suffisamment détaillées pour saisir la sémantique du domaine. À la notion d'ontologie on associe également les algorithmes permettant la traduction des concepts dans les modèles de données ainsi que les modes de représentation de ces concepts.

La définition de l'ontologie du domaine (géo-)spatial est un des grands thèmes actuels de la recherche en géomatique (citons entre autres : Frank, 1997 ; Smith et al., 1998 ; Casati et al., 1999 ; Fonseca et al., 2000). La raison essentielle est d'arriver à des définitions universelles échangeables (facilitant l'interopérabilité). La recherche concerne l'établissement des types d'objets, des processus et des relations, à différents niveaux d'échelle et de granulométrie, qui constituent le domaine (géo-)spatial (en y incorporant ou non le temps). L'ontologie spatiale se limite actuellement principalement aux outils mathématiques que sont la topologie et la méréologie (ou plus exactement une fusion des deux au travers de la méréotopologie). On se trouve dans un contexte géométrique qualitatif qui a fait l'objet de très nombreux travaux tant dans la description des objets que de leurs relations.

Notre démarche concerne exclusivement les objets géographiques discrets dans un contexte géométrique qualitatif. Nous excluons donc les phénomènes géographiques continus de notre démarche dans un premier temps. L’originalité du travail réside dans la prise en compte d'un espace projectif comme espace englobant. Cet espace, intermédiaire entre l'espace topologique et l'espace métrique (tout comme l'espace affine), a souvent été négligé dans la modélisation de l'information géographique. Nous soutenons l'idée que cette approche est utile dans la formalisation de l'ontologie spatiale. 


\section{3. Étude de la forme des objets spatiaux}

La première partie de notre démarche consiste à étudier les caractéristiques de la forme des objets spatiaux au travers de la géométrie projective ${ }^{1}$. Notons à ce stade que nous empruntons certains concepts à la géométrie affine ${ }^{2}$, la distinction entre les deux étant assez ténue. Dans la suite nous emploierons le mot projectif sauf quand la différence avec la géométrie affine est marquée. Dans ce cas, nous y ferons explicitement référence.

Cette étude n'est pas exhaustive, nous nous sommes bornés à étudier les caractéristiques qui nous paraissaient les plus intéressantes dans le contexte de l'information géographique. En premier lieu nous présentons le modèle dimensionnel qui repose sur une notion étendue de convexité. Ce modèle est un des premiers résultats aboutis de notre recherche et une importante partie de cette section lui est dédiée. Ensuite d'autres caractéristiques aux applications plus prospectives sont présentées.

\subsection{Modèle dimensionnel}

Le modèle dimensionnel (MD) a été formellement défini suivant une géométrie affine (Billen, 2002 ; Billen et al., 2002) mais peut être considéré comme projectif sous certaines conditions $^{3}$. La caractéristique essentielle de ce modèle est de proposer une segmentation sur base de critères projectifs (ou affines dans sa version générale) de la forme d'un objet spatial. Afin de bien comprendre la portée du modèle, analysons la description de la forme d'un objet suivant les différents espaces géométriques. Dans un espace géométrique basé sur la théorie des ensembles, les points formants un objet sont compris dans l'ensemble correspondant à cet objet. L'espace est donc divisé en deux parties, les points contenus dans l'ensemble et ceux qui en sont exclus. Le passage à un espace topologique permet de pousser la partition plus loin. Ne retenons dans notre raisonnement que trois primitives topologiques : l'intérieur, la frontière et l'extérieur. Ces trois primitives servent de base à plusieurs modèles topologiques de données et de relations (Egenhofer, 1991). Un point d'un objet peut donc se situer dans son intérieur topologique ou sur sa frontière topologique. Passons maintenant a un espace projectif. Les points d'un objet possèdent certaines qualités de convexité qui vont nous permettre d'envisager une autre partition. Le concept essentiel de cette partition est l'ordre d'un point d'un convexe qui est expliqué dans la section suivante.

\footnotetext{
${ }^{1}$ En termes simples, la géométrie projective concerne l'étude des figures au point de vue de leurs positions respectives et des propriétés invariantes qui les lient dans une transformation géométrique homographique (conservant l'alignement et le bi-rapport). ${ }^{2}$ La géométrie affine se différencie de la géométrie projective du fait qu'elle conserve également le parallélisme et le rapport des distances (Tisseron, 1983).

${ }^{3}$ En toute rigueur, le modèle n'est plus valide dans le cas d'une projection de tous les points à l'infini, ce qui est possible dans une géométrie projective et qui ne l'est pas en géométrie affine.
} 
À ce stade, la description de la forme d'un objet est toujours qualitative. Il est à noter que d'autres critères qualitatifs existent, certains étant reportés dans une section ultérieure. Dès que l'on passe aux espaces métriques et euclidiens, les critères deviennent quantitatifs (distance).

Le MD est largement décrit dans les références précitées. Il nous paraît néanmoins important de rappeler certains concepts afin de faciliter la compréhension du reste de l'article.

\subsubsection{Ordre d'un point d'un objet}

Chaque point d'un ensemble convexe fermé a un ordre dont la définition est donnée par BERGER (1978).

Soit $C$ un ensemble convexe dans $\boldsymbol{R}^{\mathrm{d}}$ et le point $x \in C$. L'ordre de $x$ dans $C$, noté $o(x, C)$, est la dimension de l'intersection de tous les hyperplans d'appui ${ }^{4}$ de $C$ passant par $x$. S'il n'y a pas d'hyperplan d'appui contenant $x$, alors $x$ est d'ordre $d$. On peut prouver que les points d'ordre $d$ correspondent exactement aux points intérieurs de C suivant la topologie euclidienne.

Par exemple (figure 1), par un des sommets d'un ensemble convexe fermé (un triangle dans le cas de la première figure), on peut faire passer plusieurs hyperplans d'appui. Leur intersection est un sous-espace affine de dimension 0 (un point correspondant au sommet en question). Le sommet est donc d'ordre 0 . Si on prend un point se situant sur l'arête du triangle, il n'y a qu'un hyperplan d'appui. Le sousespace affine est de dimension 1 et l'ordre de ce point sera 1 (comme tous les points de l'arête à l'exclusion des deux sommets). Enfin, pour un point se trouvant à l'intérieur, il n'y a pas d'hyperplan d'appui et l'ordre de ce point sera 2 (la dimension de l'espace englobant). D'autres exemples (goutte, ligne, cube, goutte 3D, cylindre) sont présentés dans les figures 1 et 2 .
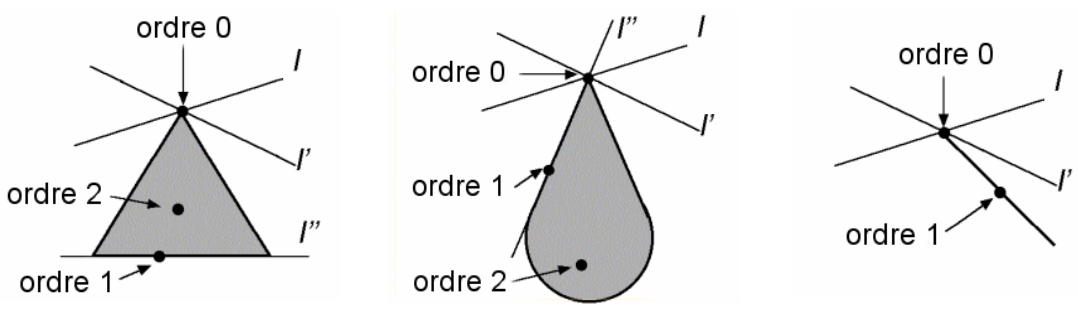

Figure 1. Ordre des points de divers objets spatiaux dans un espace $2 D$

\footnotetext{
${ }^{4}$ En termes simples, un hyperplan d'un espace $A$ de dimension d est un sous-espace de dimension (d-1) de $A$. Un hyperplan d'appui d'un ensemble convexe fermé $C$ «touche » $C$ et divise l'espace en deux parties dont l'une contient $C$. Par exemple, dans $\boldsymbol{R}^{2}$, l'hyperplan est un sous-espace de dimension 1, c'est-à-dire une ligne droite et un hyperplan d'appui de $C$ est une ligne droite $l$ tel que $C$ soit contenu dans un des demi-espaces déterminés par $l$ et tel que $l$ touche $C$.
} 

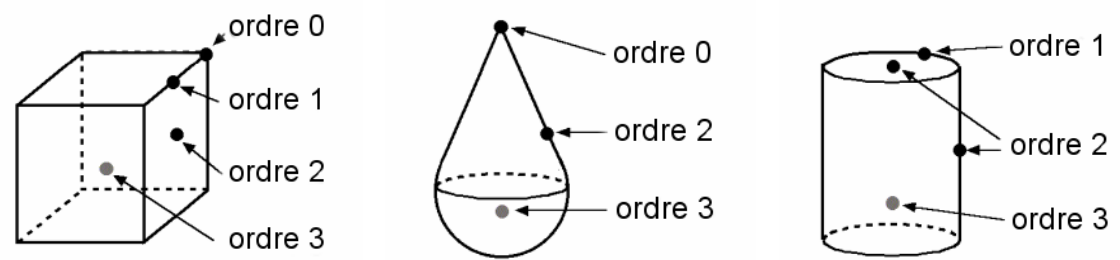

Figure 2. Ordre des points de divers objets spatiaux dans un espace $3 D$

Il est possible d'étendre ce concept à n'importe quel point d'une n-variété topologique $X$ (fermée), avec ou sans bord, dans $\boldsymbol{R}^{\mathrm{d}}(d \leq n)$, à condition qu'elle soit un sousensemble fermé de $\boldsymbol{R}^{\mathrm{d}}$. Les formules relatives à cette extension ne sont pas développées dans cet article. Le lecteur intéressé trouvera une description formelle dans Billen et al. (2002) et Billen (2002). L'important est de savoir que l'ordre des points de tous les types d'objets spatiaux peut être obtenu.

\subsection{2. Éléments dimensionnels}

Le concept d'élément dimensionnel est la base du modèle exposé tant au niveau de la définition des objets que de la définition des relations. Les éléments dimensionnels sont définis pour tous les objets spatiaux sur base de l'ordre des points.

Définition 1 : L'élément dimensionnel d'ordre $\alpha$ ( $\alpha$ D élément) d'un objet spatial $S$ (de dimension $\geq \alpha$ ) est un sous-ensemble de $S$ qui regroupe tous les points de $S$ d'ordre inférieur ou égal à $\alpha$.

L'élément dimensionnel n'a pas d'existence en dehors de l'objet dans lequel il est défini.

Définition 2 : Un élément dimensionnel d'ordre $\alpha$ ( $\alpha$ D élément) d'un objet spatial $S$ a une extension et éventuellement une limite. L'extension est le sous-ensemble de $S$ qui regroupe tous les points de $S$ d'ordre égal à $\alpha$ et la limite est le sous-ensemble de $S$ qui regroupe tous les points de $S$ d'ordre inférieur à $\alpha$. Par définition, l'élément $0 D$ n'a jamais de limite.

Remarque : Si elle existe, la limite d'un $\alpha \mathrm{D}$ élément correspond à un élément dimensionnel de dimension inférieure ${ }^{5}$.

Un exemple est présenté dans la figure 3 pour un objet simple 2D (polygone). Des cas d'éléments dimensionnels sans limite sont présentés à la figure 4.

${ }^{5}$ Cet élément n'est pas nécessairement de dimension $\alpha-1$. Il peut par exemple être de dimension $\alpha$-2. Par exemple, dans le cas d'une « goutte » (cf. figure 2), le 2D élément a pour limite un point qui est un 0D élément. 

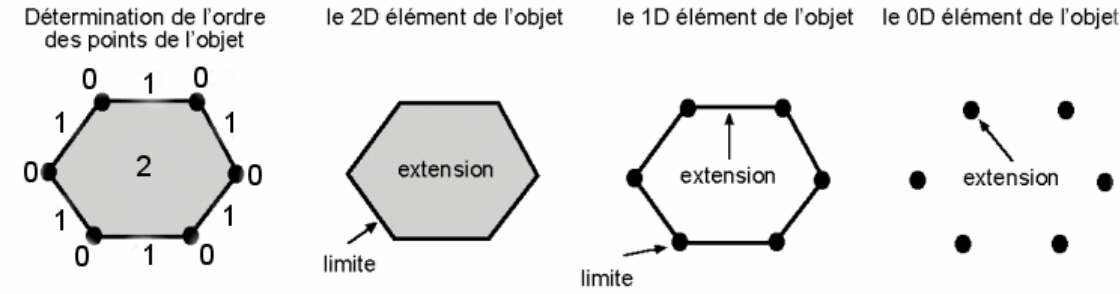

Figure 3. Éléments dimensionnels d'un polygone
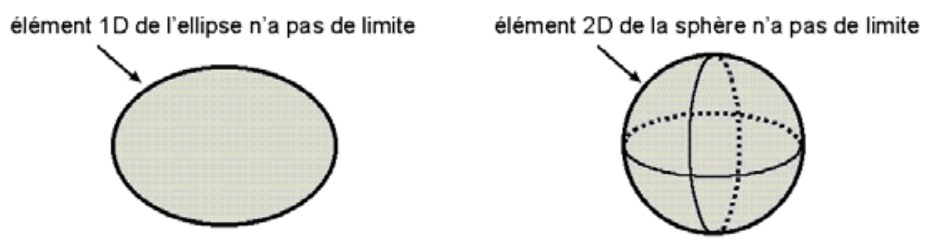

Figure 4. Éléments dimensionnels sans limite

\subsubsection{Intérêts du modèle}

Ce modèle offre une définition des objets spatiaux sur la base de nouvelles primitives (ordre et éléments). Premièrement, une classification des objets sur base de leurs éléments dimensionnels est possible. Par exemple prendre en compte la présence ou l'absence de certains éléments, ou bien le nombre de parties distinctes qui composent chaque élément. Deuxièmement, le modèle sert de base à un nouveau type de relations spatiales, les relations dimensionnelles, présentées ci-après dans la suite. Finalement, ce découpage des objets sert de base théorique au développement de modèles de données spatiales. Ces modèles comportent des primitives projectives qui lient les primitives géométriques aux objets spatiaux (Billen, 2002 ; Billen 2004).

\subsection{Autres caractéristiques projectives de la forme des objets spatiaux}

La classification des objets suivant des critères qualitatifs liés à leur forme est un sujet de recherche toujours ouvert (Galton, 2000). Notre contribution à cette problématique, outre la définition du modèle dimensionnel, réside en une tentative de lier expressément certains critères qualitatifs à une théorie mathématique formelle. Le concept d'ordre d'un point d'un convexe n'est pas la seule caractéristique projective 
qui peut être utilisée dans la description de la forme des objets spatiaux. Plusieurs auteurs se sont déjà intéressés à la notion de concavité d’un objet. La détermination des concavités d'un objet peut se faire de plusieurs manières ; soit en étudiant le signe des rayons de courbure et les points d'inflexion, soit en servant de la notion d'enveloppe convexe ${ }^{6}$ d'un objet (Cohn, 1995). Par définition, si $B$ est un sousensemble de $\boldsymbol{R}^{\mathrm{d}}$, l'enveloppe convexe de $B$, notée $\operatorname{conv}(B)$, est le plus petit ensemble convexe de $\boldsymbol{R}^{\mathrm{d}}$ contenant $B$. Il est possible d'obtenir les concavités d'un objet en soustrayant l'objet à son enveloppe convexe. Une hiérarchie des concavités peut être trouvée en appliquant récursivement cette opération aux différentes parties de concavités (qui peuvent être isolées par méréologie) (figure 5).

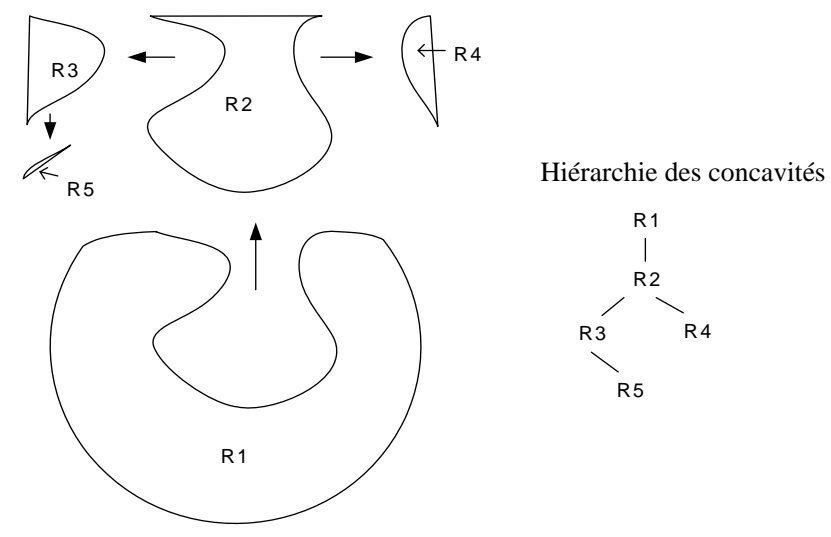

Figure 5. Hiérarchie des concavités d'une région

À ce stade, une classification qualitative des objets est possible en regardant l'ordre des points (absence ou présence de points d'ordre 0 par exemple) (figure 6), les concavités (absence, présence, hiérarchie). Mais, nous pouvons avancer de façon prospective que d'autres caractéristiques peuvent être utilisées, comme le signe du rayon de courbure $(-, 0,+)$, le type de concavité, le concept d'objet étoilé qui sont toujours des caractéristiques projectives, ou, en passant à une géométrie affine, l'étude de la position relative du barycentre par rapport à l'objet. Illustrons notre propos en introduisant les deux derniers concepts.

Le concept d'objet étoilé mérite une explication. Un ensemble $S$ est dit étoilé si par un de ses points $x$ il est possible de tracer des segments, inclus dans $S$, avec tous les autres points de $S$. En d'autres termes, s'il existe un point qui permet d'atteindre tous les points de la surface de l'objet sans croiser le contour. Un objet convexe est toujours étoilé. Dans le cas d'un objet concave, on peut regarder si la région est étoilée

${ }^{6}$ L'enveloppe convexe d'un objet est le plus petit ensemble convexe qui contient cet objet. 
ou non, mais également appliquer cette propriété à ses concavités, ainsi qu’à l'extérieur de la région en considérant des segments connectés à l'infini (figure 7). On arriverait, par exemple, à distinguer les formes présentées à la figure 8.

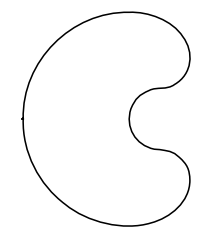

$\#$ ordre $0=0$

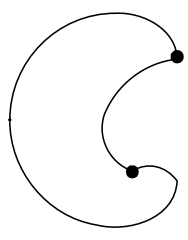

\# ordre $0=2$

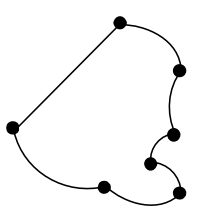

$\#$ ordre $0=7$

Figure 6. Différentes régions selon le nombre de points d'ordre 0
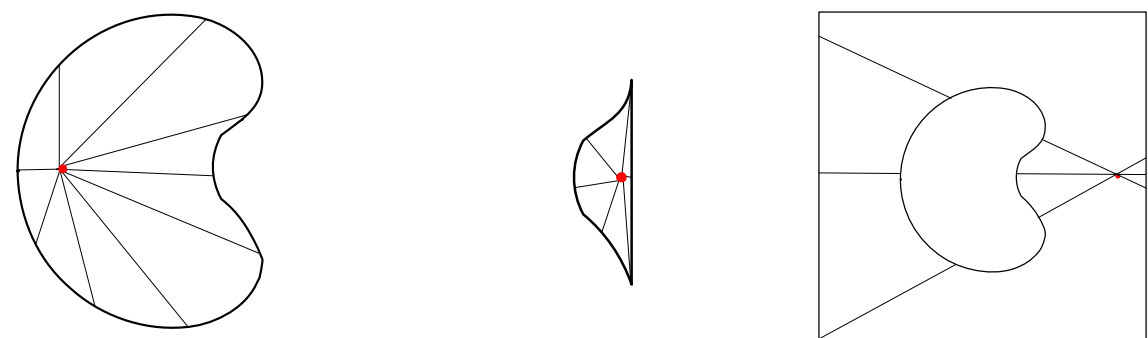

Figure 7. Régions étoilées (objet et concavité) et extérieur étoilé
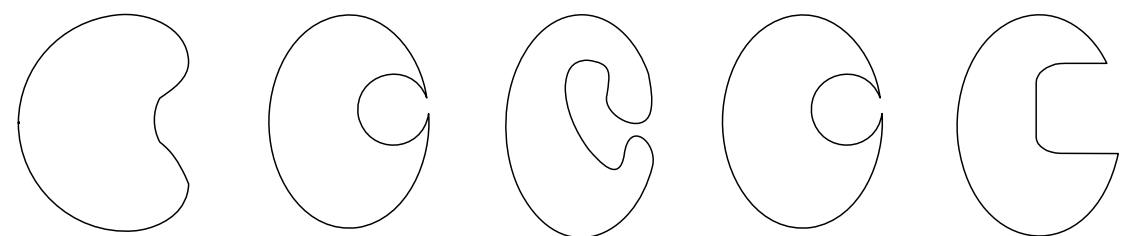

Figure 8. Objets distinguables suivant le concept de région étoilée

Le barycentre d'un objet peut se trouver dans l'objet, sur sa frontière ou à l'extérieur. L'étude de la position du barycentre en conjonction avec une étude sur les concavités peut amener à une certaine distinction qualitative de la forme des objets. On arrive à des situations où l'on peut estimer qualitativement la " profondeur » de concavité de l'objet. Dans le premier exemple (figure 9), la concavité est "peu profonde », dans l'autre elle est « assez profonde ». L'emploi de ces termes est intuitif et ne repose sur aucune étude spécifique. 

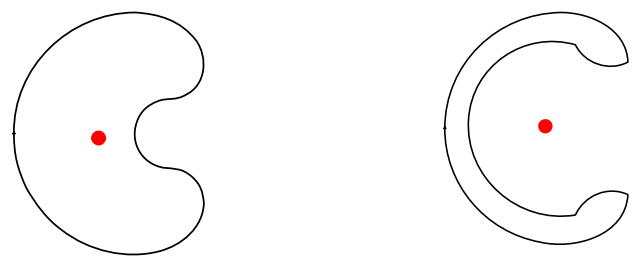

Figure 9. Distinction qualitative de concavités par l'étude de la position du barycentre

En étudiant les caractéristiques projectives et affines de la forme des objets, on obtient une base intéressante en vue d'une catégorisation qualitative des objets. Leur caractère formel d'un point de vue mathématique en fait de bons candidats pour l'analyse spatiale.

\section{Relations projectives binaires}

De nombreuses relations spatiales sont décrites sur la base de relations binaires, c'est-à-dire mettant en jeu deux objets. Les relations topologiques par exemple suivent cette approche. Avant de présenter les relations projectives binaires issues de notre recherche, il est bon de repositionner le problème des relations topologiques. Le principe général est simple. Il repose sur la comparaison d'ensembles de points. Un objet peut être vu comme un ensemble de points dans un univers. Les relations possibles entre deux objets sont déduites des relations entre ensembles, à savoir, l'intersection, l'inclusion, la contenance et l'égalité. Les modèles de relations topologiques suivent cette démarche appliquée à ses composants ou primitives topologiques. La topologie permet de dissocier l'objet en différentes parties, et la relation spatiale est déduite des relations entre les sous-ensembles de points de l'objet que sont les primitives topologiques. La démarche concernant les relations binaires projectives est la même. L'objet, originellement un ensemble de points est segmenté en sous-ensembles suivant des critères projectifs. Ce sont ces sous-ensembles qui sont ensuite comparés.

\subsection{Relations dimensionnelles}

Par définition, une relation dimensionnelle est une relation qui décrit l'intersection entre éléments dimensionnels sur base de leurs extensions et de leurs limites. Notons que la même catégorisation de relations peut être obtenue en travaillant sur un groupement plus simple des points en fonction de leur ordre (sous-ensembles de points d'un ordre donné, qui correspond à l'extension de l'élément dimensionnel d'ordre correspondant). Le modèle évoluerait vers un développement plus proche de 
ce qui existe pour les relations topologiques, en se basant sur l'étude des intersections entre les sous-ensembles, en incluant une notion d'extérieur (identique à l'extérieur topologique). Ce choix n’a pas été fait, et nous présentons succinctement les règles qui ont été développées.

Les relations dimensionnelles peuvent être totales, partielles ou nulles.

Définition 1 : Un élément dimensionnel est en relation dimensionnelle totale avec un autre élément dimensionnel si leur intersection est égale au premier élément et si l'intersection entre leurs extensions n'est pas vide.

Définition 2 : Un élément dimensionnel est en relation dimensionnelle partielle avec un autre élément dimensionnel si leur intersection n'est pas égale au premier élément et si l'intersection entre leurs extensions n'est pas vide.

Définition 3 : Un élément dimensionnel est en relation dimensionnelle nulle avec un autre élément dimensionnel si l'intersection entre leurs extensions est vide.

Les différents types de relations dimensionnelles peuvent être affinés, en prenant en compte : le type d'éléments dimensionnels ; la dimension de l’intersection ; le nombre de parties de l'intersection. On arrive ainsi à la description complète de la relation dimensionnelle entre deux éléments dimensionnels.

Relation dimensionnelle : $(\operatorname{dim} A, \operatorname{dim} B$, type, $\operatorname{dim} i n t, n b$ int $)$

$\operatorname{Dim} A$ : dimension du premier élément dimensionnel, $(0, . ., n)$.

$\operatorname{Dim} B$ : dimension du deuxième élément dimensionnel, $(0, . ., \mathrm{n})$.

Type : type de relation (nulle, totale, partielle).

Dim int : dimension de l'intersection, $(\varnothing, 0, . ., n)$.

$N b$ int : nombre de parties distinctes de l'intersection, $(0, . ., N)$, a étant le nombre de parties distinctes du premier élément dimensionnel.

\begin{tabular}{|c|c|c|c|c|c|}
\hline Dim A & Dim B & Type & Dim int & Nb int & Exemple \\
\hline 1 & 2 & Nulle & $\varnothing$ & 0 & - \\
\hline 1 & 2 & Totale & 1 & $a$ & \\
\hline 1 & 2 & Partielle & 1 & $N$ & \\
\hline & & & & & \\
\hline
\end{tabular}

Tableau 1. Ordre des points de divers objets spatiaux (dans un espace 2D et 3D) 
Le tableau 1 présente les relations dimensionnelles possibles entre un 1D élément d'un objet et un 2D élément d'un autre objet. La liste complète des relations dimensionnelles possibles est dressée dans Billen (2002).

Sur base de ces relations dimensionnelles, on peut définir de nouvelles relations spatiales. La démarche comprend trois étapes :

1. On identifie les éléments dimensionnels des deux objets.

2. On identifie tous les couples potentiels d'éléments dimensionnels des deux objets.

3. On établit la relation dimensionnelle pour chaque couple.

On aboutit ainsi à une liste de relations qui décrit une configuration spatiale particulière sur base du critère projectif d'ordre d'un point. Le nombre de relations spatiales ainsi détectées est fini. On obtient des groupements de relations correspondant à des équivalences projectives. Les figures suivantes montrent quelques exemples de relations spatiales équivalentes d'un point de vue topologique ${ }^{7}$, qui sont différenciées par relations dimensionnelles. On voit qu'il est possible de différencier une intersection entre deux polygones selon le nombre de points d'ordre 0 prenant part à la relation (figure 10) ainsi que le cas ou un cube est en contact en un point avec un autre cube suivant un de ses coins (ordre 0 ), une de ses arêtes (ordre 1 ) ou une de ses faces (ordre 2) (figure 11).

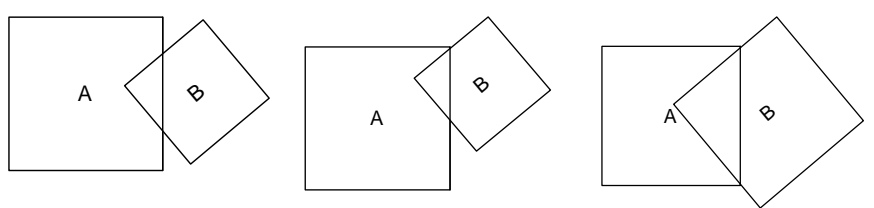

Figure 10. Équivalences topologiques distinguables par relations dimensionnelles - 1
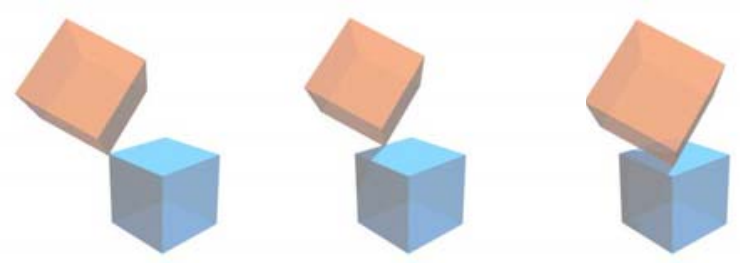

Figure 11. Équivalences topologiques distinguables par relations dimensionnelles - 2

\footnotetext{
${ }^{7}$ Les relations dimensionnelles ne sont pas à proprement parler une extension des relations topologiques (vu qu'elles se basent sur un autre espace mathématique). Cependant des correspondances existent qui nous permettent d'illustrer la résolution de certaines équivalences topologiques. L'établissement de ces correspondances sort du cadre de ce travail et sont présentées dans Billen (2002).
} 


\subsection{Intérêts des relations dimensionnelles}

L'obtention d'une catégorie plus complexe de relations spatiales a été identifiée comme importante, surtout en analyse spatiale 3D, notamment par Breunig (1996). Le développement proposé par cet auteur est lié au mode de représentation choisi (une variation d'un 2-complex simplicial ${ }^{8}$ dans ce cas). Il propose de prendre en compte le type de simplexe en jeu dans les relations topologiques afin d'opérer une distinction de ces relations. Les relations particulières obtenues sont illustrées dans la figure 12.
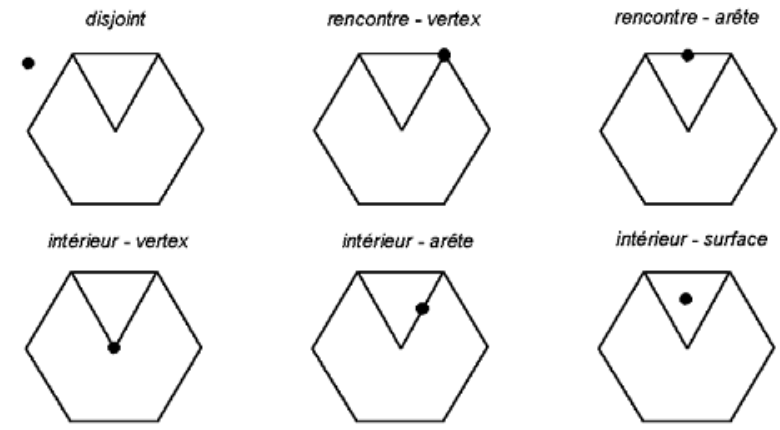

Figure 12. Raffinement des relations topologiques suivant Breunig

Mais ce type de relations ne correspond pas à des caractéristiques intrinsèques des objets, mais à leur mode de représentation. Ainsi, dans l'exemple suivant (figure 13), le choix de la triangulation d'un carré induit un changement dans la relation entre le point et le carré, alors qu'ils se trouvent exactement dans la même position.
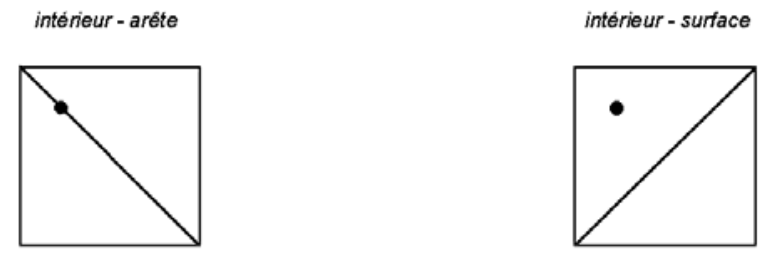

Figure 13. Le raffinement est dépendant du mode de représentation

\footnotetext{
${ }^{8}$ Pour rappel, les éléments les plus simples de l'espace euclidien (en se limitant à la dimension 3) pour une dimension donnée sont le point, le segment de droite, le triangle et le tétraèdre. Ces éléments sont appelés simplexes, le point est le 0simplexe, le segment de droite le 1-simplexe et ainsi de suite. Les complexes simpliciaux sont obtenus en «collant» des simplexes le long de leurs faces suivant certaines règles que nous n'aborderons pas dans ce travail. Des descriptions détaillées peuvent être trouvées notamment dans Breunig (1996) et Billen (2002).
} 
Notre approche, étant basée sur des caractéristiques projectives des objets, ne rencontre pas ce genre de problème et permet une description qualitative des relations spatiales.

\section{Relations ternaires}

Bien que faisant l'objet de plusieurs travaux (Freska, 1992 ; Scivos et al., 2001 ; Favetta, 2003) les relations ternaires, relations entre trois objets, sont relativement marginales en sciences de l'information géographique.

L'originalité de notre démarche consiste en la prise en considération d'une segmentation projective de l'espace sur base de deux objets de référence (Billen et al., 2004). A l'heure actuelle, seules les relations entre points et entre régions dans un espace à deux dimensions ont été développées. L'extension du modèle aux lignes et aux cas mixtes en 2D ainsi que l'extension du modèle complet en 3D sont également prévues.

Le principe de notre modèle est de décomposer l'espace en cinq parties sur base de propriétés projectives de deux objets. A ces partitions invariantes suivant des transformations projectives nous avons associé des mots du langage courant qui nous paraissent transcrire les caractéristiques de ces partitions. Ces mots sont avant, entre, après, à gauche de, à droite de.

\subsection{Relations ternaires entre points}

Le modèle de relations ternaires entre points présente quelques similitudes avec l'approche de Freska (1992). Considérant deux points de référence, il est possible de diviser l'espace en cinq parties qui sont présentées à la figure 14a. Ces cinq parties correspondent en fait au niveau inférieur d'une hiérarchie de relations projectives ternaires (figure 14b).

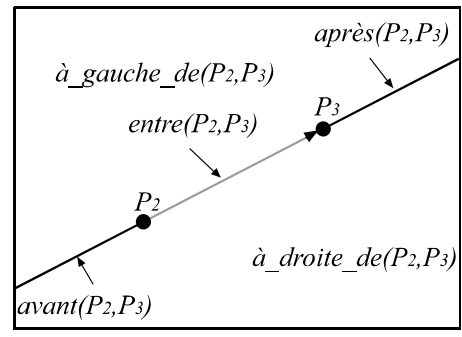

a. Partition projective du plan

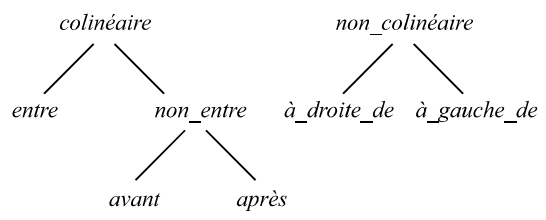

b. Modèle hiérarchique des relations

Figure 14. Relations projectives entre trois points 
Les définitions formelles de ces relations ont été présentées dans des travaux ultérieurs et ne sont pas reproduites dans cet article. L'interprétation de ces relations est néanmoins assez intuitive. Un point P1 se situera dans une des cinq parties de l'espace formées par les points de référence P2 et P3. Les notations suivantes ont été introduites pour refléter ces relations: $\operatorname{avant}\left(P_{1}, P_{2}, P_{3}\right)$, entre $\left(P_{1}, P_{2}, P_{3}\right)$, après $\left(P_{1}, P_{2}, P_{3}\right)$, à_droite_de $\left(P_{1}, P_{2}, P_{3}\right)$, à ${ }_{-}$gauche_de $\left(P_{1}, P_{2}, P_{3}\right)$.

\subsection{Relations ternaires entre régions}

L'extension des relations projectives ternaires entre régions n'est pas aussi directe. Il est nécessaire de regarder plus en détails la définition des relations afin de bien appréhender le reste de l'article. Les définitions formelles du modèle sont développées dans un autre travail (Billen et al., 2004).

Le modèle hiérarchique des relations projectives entre régions est similaire à celui présenté pour les points. Dans la suite, on indique l'enveloppe convexe d'une région par une fonction $\mathrm{CH}()$ ( $\mathrm{CH}$ pour Convex Hull). La partition projective de l'espace en cinq régions correspondantes aux cinq relations projectives est basée sur la définition de la relation générale de colinéarité entre trois régions. La portion d'espace où cette relation est vraie est appelée la région de colinéarité et est délimitée par quatre lignes qui sont les tangentes communes externes et les tangentes communes internes. Les tangentes communes externes de B et C sont définies par le fait qu'elles sont aussi tangentes à l'enveloppe convexe de l'union de $\mathrm{B}$ et $\mathrm{C}$ (figure 15a). Les tangentes communes internes se croisent à l'intérieur de l'enveloppe convexe de l'union de $\mathrm{B}$ et C et divisent le plan en quatre cônes (figure 15b). Afin de distinguer ces quatre cônes, on considère une ligne orientée de la région $B$ à la région $C$ et on appelle Cone $_{-\infty}(B, C)$ le cône qui contient la région $\mathrm{B}$, Cone $_{+\infty}(B, C)$ le cône qui contient la région $\mathrm{C}$, $C$ one $e^{+}(B, C)$ le cône qui est à la droite de la ligne orientée, $C{ }^{-}(B, C)$ le cône qui est à la gauche de la ligne orientée.

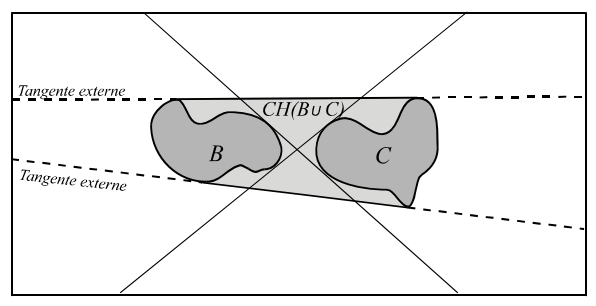

a. Tangentes communes externes

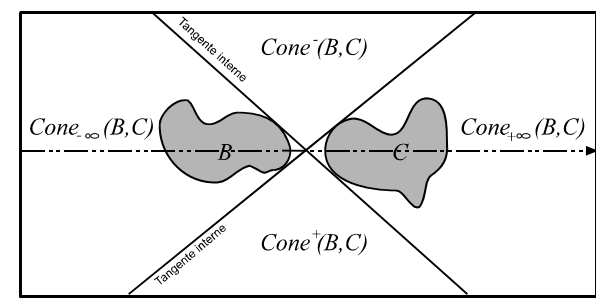

b. Tangentes communes internes

Figure 15. Tangentes communes de deux régions $B$ et $C$

On obtient une partition de l'espace en cinq régions qui correspondent aux cinq relations projectives de base: avant, entre, après, à gauche de, à droite de. Par souci 
de simplicité, donnons un nom aux régions du plan correspondant aux relations basiques :
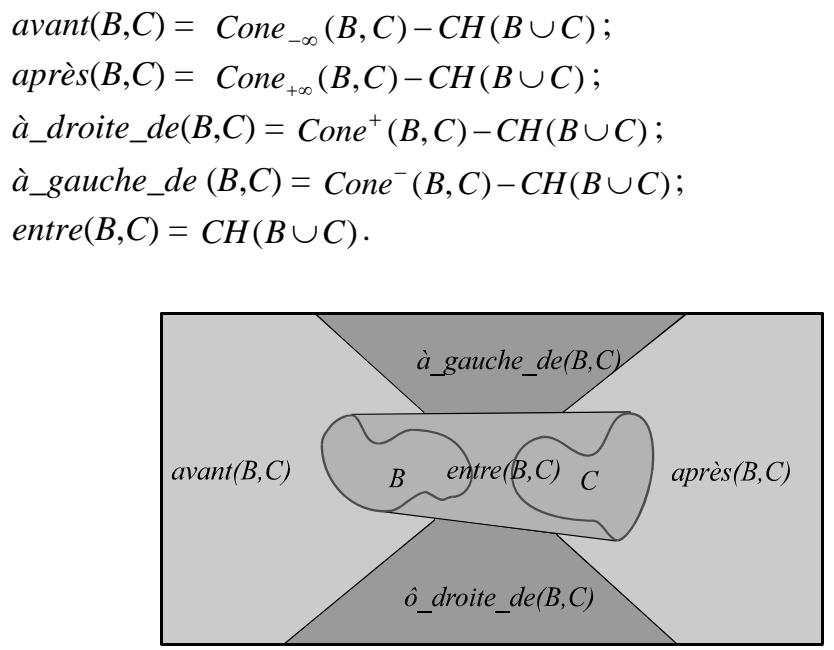

Figure 16. Partition projective de l'espace en cinq régions

L'ensemble des cinq relations projectives peut être utilisé comme un ensemble de relations de base afin de construire un modèle pour toutes les relations projectives ternaires.

Le modèle est appelé le modèle des 5-intersections et peut être exprimé par une matrice de cinq valeurs qui sont les intersections vides (valeur 0) / non vides (valeur 1) de la région $A$ avec les cinq régions définies ci-avant (figure 17). Les cinq relations de base correspondent aux valeurs de la matrice avec seulement une intersection non vide (figure 18). Au total, la matrice des 5 -intersections peut avoir $2^{5}$ valeurs différentes qui correspondent au nombre théorique de relations projectives. En réalité, seules 31 relations projectives différentes entre les trois régions $A, B$ et $C$ existent dans un espace à deux dimensions.

\begin{tabular}{|c|c|c|}
\hline & $\begin{array}{c}A \cap \\
\text { à_gauche_de }(B, C)\end{array}$ & \\
\hline $\begin{array}{c}A \cap \\
\operatorname{avant}(B, C)\end{array}$ & $\begin{array}{c}A \cap \\
\text { entre }(B, C)\end{array}$ & $\begin{array}{c}A \cap \\
\operatorname{après}(B, C)\end{array}$ \\
\hline & $\begin{array}{c}A \cap \\
\text { à_droite_de }(B, C)\end{array}$ & \\
\hline
\end{tabular}

Figure 17. Matrice des 5-intersections 


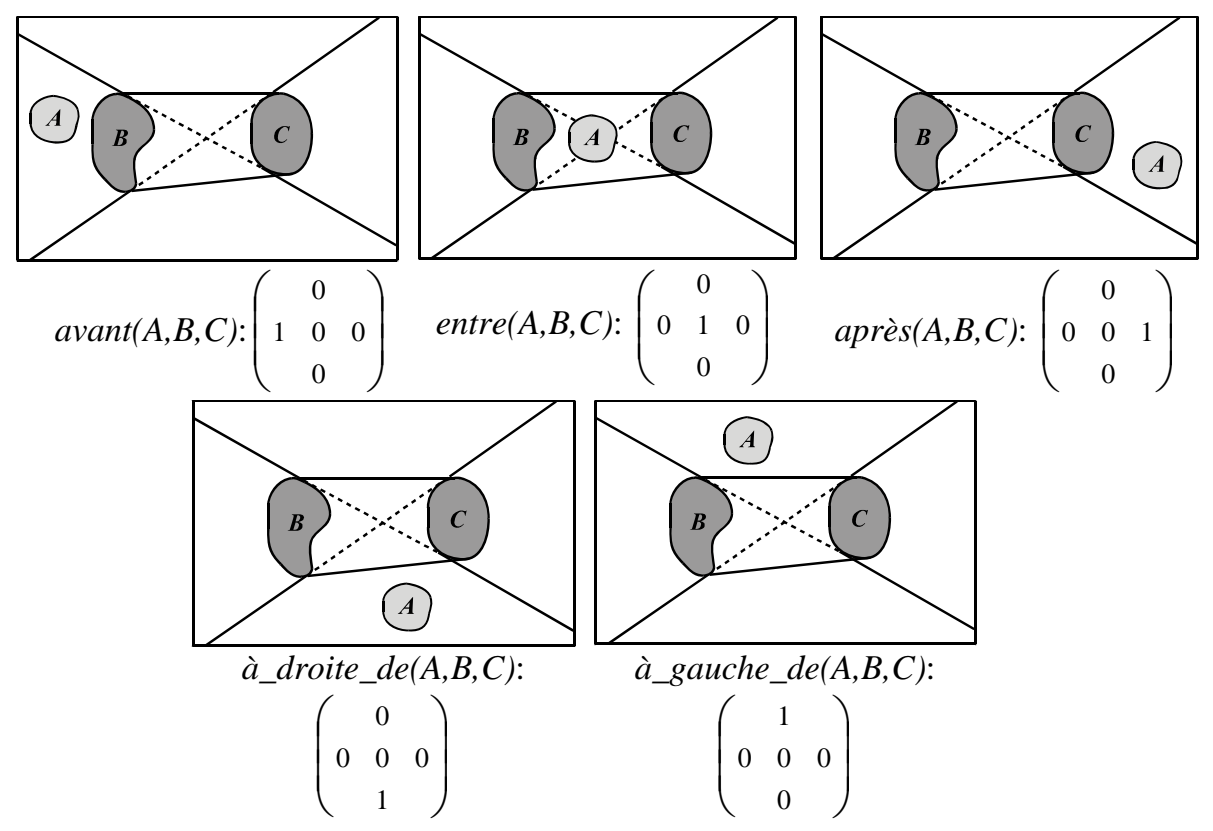

Figure 18. Les cinq relations ternaires de base entre trois régions $A, B$ et $C$

\subsection{Intérêts des relations ternaires}

La correspondance des relations avec notre perception du monde réel paraît claire même si elle n’a pas été démontrée actuellement. La pertinence de l'espace projectif comme espace englobant l'est aussi. Prenons le cas d'un vol en avion au-dessus d'une contrée. L'observateur perçoit une scène dont il ne connaît aucune composante métrique. Mais, il peut déduire des observations « projectives » du type « la ville A se situe à gauche de la ville $\mathrm{B}$ et $\mathrm{C} »$. Ces relations seront maintenues quel que soit son point de vue (jusqu'au cas limite où tous les objets se trouvent dans le même plan de vision).

La perception naturelle des notions à gauche de, à droite de, etc. est peut être un peu différente. Mais il est concevable de l'expliquer avec notre modèle. En effet, un être humain perçoit ces notions, et peut positionner n'importe quel objet par rapport à son propre référentiel. Néanmoins, on ne se trouve pas dans le cas d'une relation entre deux objets, mais bien d'une relation entre un objet et un objet de référence orienté. Cette orientation correspond en réalité à un autre objet de référence implicite. Si on considère la perception des cinq régions projectives qu'à un observateur, on peut envisager plusieurs cas de figure (dans la figure 19, l'observateur est vu du dessus, ses yeux pointant vers la droite de la feuille). Les deux objets de référence sont-ils deux 
points du crane de l'observateur (premier schéma) ou bien une ligne (joignant les deux yeux) et un point postérieur (deuxième schéma) ? Ou bien encore deux lignes (troisième schéma) ? En tous les cas il serait intéressant de confronter les relations qui découleraient de notre théorie formelle avec une étude de type géographie naïve.
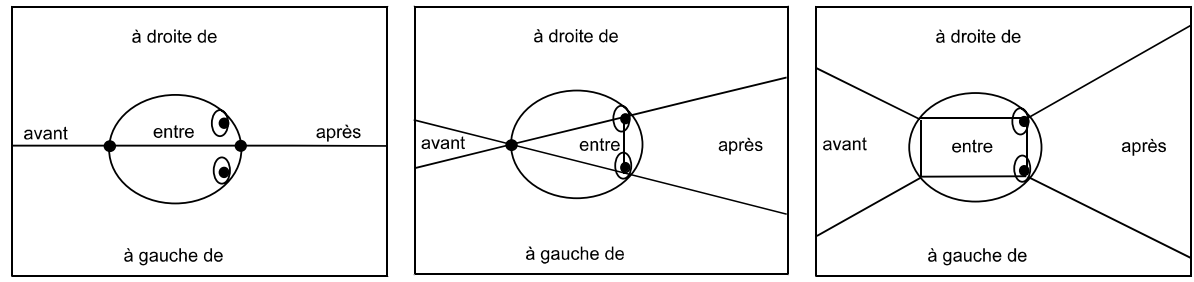

Figure 19. Tentative d'application du modèle des 5-intersections à un observateur

\section{Conclusions}

Dans cet article, nous avons montré que la prise en compte de caractéristiques projectives des objets spatiaux et de leurs relations aboutit à la définition formelle de plusieurs concepts utiles dans la définition de l'ontologie spatiale.

La segmentation projective des objets spatiaux offre des possibilités intéressantes en termes de modèles de données et sert de base à un nouveau type de relations spatiales. De plus, en considérant d'autres critères de forme tels les concavités des objets, on peut obtenir une catégorisation qualitative des objets spatiaux. Le nouveau type de relations spatiales binaires, les relations dimensionnelles, offre la possibilité de distinguer des configurations spatiales assez complexes, telles que «toucher le (coin / arête / face) d'un objet », le tout dans un cadre formel basé sur des définitions mathématiques strictes. Finalement, les relations projectives ternaires représentent une véritable innovation qui permet de décrire des relations spatiales telles que « être à la gauche de tel et tel objets » « être devant tel et tel objets ».

Les travaux entrepris jusqu'à présent sont loin d'exploiter toute les potentialités des espaces intermédiaires entre l'espace topologique et l'espace métrique. Le champ d'investigation est encore très large et doit permettre à l'avenir de définir formellement d'autres relations et concepts utiles dans la définition d'ontologies spatiales. 
Ce travail a été supporte par M.I.U.R. (Ministère italien de l'éducation et de la recherche) à travers le projet "Representation and management of spatial and geographic data on the Web”.

\section{Bibliographie}

Berger M., Géométrie 3 : convexes et polytopes, polyèdres réguliers, aires et volumes, Paris, Cedic/Fernand Nathan, 1978.

Billen R., Nouvelle perception de la spatialité des objets et de leurs relations - Développement d'une modélisation tridimensionnelle de l'information spatiale, Thèse de doctorat, Université de Liège, 2002.

Billen R., « A projective approach to handle 3D spatial data », Proceedings of GIS Research UK 2004, University of East Anglia, Norwich, 28-30 April 2004, Angleterre, pp. 237-240.

Billen R., Clementini E., «A model for ternary projective relations between regions », Advances in Database Technology - EDBT 2004: 9th International Conference on Extending Database Technology, Heraklion, Crete, Greece, March 14-18, 2004, Lecture notes in computer science, Springer-Verlag, Heidelberg, pp. 310-328.

Billen R., Zlatanova S., Mathonet P., Boniver F., « The Dimensional Model: a framework to distinguish spatial relationships ", Advances in Spatial Data Handling, Richardson D. et Van Oosterom P., (eds), Heidelberg, Springer-Verlag, 2002, pp. 285-298.

Breunig M., Integration of Spatial Information for Geo-Information Systems, Lecture Notes in Earth Sciences 61, Berlin, Springer-Verlag, 1996.

Casati R., Varzi A., Parts and Places: The structures of spatial representations, Cambridge, MA: The MIT Press, 1999.

Clementini E., Di Felice P., « Spatial Operators », ACM SIGMOD Record, vol. 29(3), 2000, p. 31-38.

Clementini E., Di Felice P., Hernández D., «Qualitative representation of positional information », Artificial Intelligence, vol. 95, 1997, p. 317-356.

Clementini E., Di Felice P., van Oosterom P., «A Small Set of Formal Topological Relationships Suitable for End-User Interaction », Advances in Spatial Databases - Third International Symposium, SSD '93, Abel D., et Ooi B., (eds), Berlin, Springer-Verlag, 1993, p. 277-295.

Cohn, A., «A hierarchical representation of qualitative shape based on connection and convexity ", Spatial Information Theory: A Theoretical Basis for GIS - International Conference, COSIT'95, Frank A., et Kuhn W., (eds), Berlin, Springer-Verlag, 1995, p. 311326.

Cui, Z., Cohn, A. et al. (1993). «Qualitative and Topological Relationships in Spatial Databases», Advances in Spatial Databases - Third International Symposium, SSD '93, , Abel D., et Ooi B., (eds), Berlin, Springer-Verlag, 1993, p. 296-315. 
Egenhofer M., Herring J., « Categorizing Binary Topological Relationships Between Regions, Lines, and Points in Geographic Databases », Department of Surveying Engineering, University of Maine, Orono, ME, 1999.

Favetta, F., Ambiguïtés dans les langages visuels d'interrogation spatiale et précision topologique des Bases de Données spatiales, Thèse de doctorat, INSA-Lyon, 2003

Fonseca F., Egenhofer M., Davis C., Borges K., « Ontologies and Knowledge Sharing in Urban GIS », Computer, Environment and Urban Systems, Vol. 24(3), 2000.

Frank A., « Ontology A Geographical Point of View », Spatial and Temporal Reasoning, Stock O., (éd.), Dordrecht, The Netherlands, Kluwer Academic Publishers, 1997, p. 135-153.

Freksa C., " Using Orientation Information for Qualitative Spatial Reasoning », Theories and Models of Spatio-Temporal Reasoning in Geographic Space, Frank A., Campari I., Formentini U., (eds), Berlin, Springer-Verlag, 1992, p. 162-178.

Galton A., Qualitative Spatial Change, Oxford, Oxford University Press, 2000.

Gapp K.-P., " Angle, Distance, Shape, and their Relationship to Projective Relations » Proceedings of the 17th Conference of the Cognitive Science Society, Pittsburgh, PA, 1995.

Hayes P., "The naive physices manifesto », Expert Systems in the Microelectronic Age, Mitchie D., (éd), Edinburgh, Edinburgh University Press, 1978, p. 242-270.

Hernández D., Qualitative Representation of Spatial Knowledge, Lecture Notes in Artificial Intelligence, Vol. LNAI 804, 1994, Berlin, Springer-Verlag.

Scivos A., Nebel B., « Double-Crossing: Decidability and Computational Complexity of a Qualitative Calculus for Navigation ", Spatial Information Theory. Foundations of Geographic Information Science: International Conference, COSIT 2001, Montello D., (éd), Springer, 2001 p. 431-446.

Smith B., Mark D., " Ontology and Geographic Kinds », Proceedings of the International Symposium on Spatial Data Handling (SDH'98), Vancouver, Cananda, 1998.

Tisseron C., Géométrie affine, projective et euclidienne, Paris, Hermann, 1983.

Vorwerg C., « Projective relations for 3D space: Computational model, application, and psychological evaluation ", Proceedings of the 14th National Conference on Artificial Intelligence and 9th Innovative Applications of Artificial Intelligence Conference, AAAI 97, IAAI 97, Providence, Rhode Island, AAAI Press / The MIT Press, 1997, p. 159-164. 